\title{
Clinical Trial Report: The Presence and Rupture of Vulnerable Plaques in the Peripheral Circulation
}

\author{
Anand Prasad $\cdot$ Sotirios Tsimikas
}

Published online: 8 June 2010

(C) The Author(s) 2010. This article is published with open access at Springerlink.com

Okura H, Asawa $\mathrm{K}$, Kubo $\mathrm{T}$, et al.: Incidence and predictors of plaque rupture in the peripheral arteries. Circ Cardiovasc Interv 2010, 3:63-70.

\section{Rating \\ •• Of major importance.}

\section{Introduction}

The incidence, associated risk factors, and clinical consequences of coronary artery plaque rupture have been previously well described $[1,2]$. In contrast, similar data for the peripheral vasculature are notably lacking. Okura et al. investigate the prevalence of plaque rupture in the iliofemoral circulation by intravascular ultrasound (IVUS). The authors examine the relationship between the presence of plaque rupture and patient characteristics and correlate their findings with clinical outcomes.
Aim
A. Prasad $\cdot$ S. Tsimikas $(\bowtie)$
University of California San Diego,
9500 Gilman Drive, BSB-1080,
La Jolla, CA 92093-0682, USA
e-mail: stsimikas@ad.ucsd.edu

The authors intended to define the prevalence and clinical characteristics of plaque rupture by IVUS in patients with peripheral arterial disease (PAD) undergoing percutaneous intervention of ilio-femoral atherosclerotic lesions.

\section{Methods}

The study examined 101 patients (with a total of 101 lesions: 28 common iliac, 27 external iliac, and 46 superficial femoral arteries) undergoing clinically indicated peripheral angioplasty. All patients had hsCRP levels measured the day prior to the procedure. Angiography and IVUS (using instruments from three different manufacturers) using automated pullback at $1 \mathrm{~mm} / \mathrm{sec}$ were performed to image the entirety of each respective arterial conduit. The contralateral vessel was not imaged. Standard IVUS dimensions were measured, including luminal crosssectional area (CSA), vessel area, external elastic membrane (EEM) area, and plaque burden. Plaque rupture was defined as the presence of a cavity that communicated with the lumen with an overlying residual fibrous cap fragment. Clinical outcomes including death, myocardial infarction, unplanned peripheral arterial revascularization (angioplasty or bypass surgery), ischemic stroke, and amputation (major or minor) were followed prospectively.

\section{Results}

Forty-two patients (42\%) had evidence of plaque rupture, with the majority of individuals having a single rupture (26 patients). The two groups (with vs without plaque rupture) were both similar in terms of age, ankle brachial index, and Fontaine class. However, the patients with plaque rupture in the ilio-femoral arteries more often presented with a history of acute coronary syndromes (ACS; $43 \%$ vs $17 \%, P=0.004$ ). Target lesion plaque ulceration by angiography was associated with rupture by IVUS $(P<0.0001)$. Target lesion EEM CSA, lumen CSA, and plaque plus media CSA were significantly larger in 
patients with plaque rupture than in patients without plaque rupture. By multivariable logistic regression analysis, both history of $\mathrm{ACS}\left(\chi^{2}=8.1\right.$, odds ratio $[\mathrm{OR}]=4.1,95 \% \mathrm{CI}=$ $1.55-10.80, P=0.004)$ and male sex $\left(\chi^{2}=6.1, \mathrm{OR}=4.8\right.$, $95 \% \mathrm{CI}=1.39-16.39, P=0.01)$ were independent clinical correlates of plaque rupture in the ilio-femoral arteries. During follow-up (median, 14.7 months), the incidence of major adverse cardiac or cerebrovascular events (death, myocardial infarction, and ischemic stroke [MACCE]) was similar between the two groups. However, the incidence of MACCE plus peripheral vascular events (unplanned vascular intervention and amputation) was significantly higher in patients with plaque rupture than in patients without plaque rupture $(46 \%$ vs $21 \%, P=0.008)$. By multivariable Cox regression analysis, plaque rupture (hazard ratio $=2.80,95 \% \mathrm{CI}=1.23-6.37, P=0.01$ ) and Fontaine stage IV (hazard ratio $=3.50,95 \% \mathrm{CI}=1.58$ 7.71, $P=0.002$ ) were independent predictors of MACCE plus peripheral vascular events.

\section{Discussion}

The authors examine the prevalence and clinical significance of plaque rupture in the ileo-femoral circulation. In these patients undergoing peripheral angioplasty, plaque rupture was noted in $42 \%$ of patients, suggesting that this is not a rare finding in patients with symptomatic PAD. Two key parallels are noted in this study between peripheral plaque rupture and data from prior coronary atheroma plaque studies [3]. First, luminal cross-sectional area and EEM areas were larger at the target lesion in the patients who had plaque rupture versus those without rupture, suggesting that the process of positive remodeling is similar in peripheral arteries as in the coronary arteries. Second, patients who had plaque rupture in the periphery were more likely to have had ACS, consistent with the concept that atherosclerosis is a systemic disease. These data linking plaque vulnerability in multiple circulations in high-risk patients are hypothesis generating. Although the sample size is small (101 patients/arteries), this article represents the largest evaluation of this phenomenon.

\section{Comments}

The presence of fibrous and fibrocalcific atheromas has been well described in the peripheral circulation [4-6]. Although less commonly reported, lipid-laden atherosclerotic lesions with thin fibrous caps have also been demonstrated. Pasterkamp et al. [7] examined the inflammatory characteristics of thin-capped fibroatheromas in the femoral arteries taken at autopsy from patients who died from non-cardiovascular causes. Even in these "low-risk" patients, vulnerable plaques were identified in the peripheral circulation. In 50 arteries, the authors documented heavy or moderate staining for macrophages in the cap or shoulder region in $45 \%$ of femoral artery atheromas. Furthermore, those lesions that had evidence of plaque rupture demonstrated evidence of macrophage staining. These results are similar to the large body of data linking inflammation with vulnerable plaque formation and rupture in the coronary circulation.

The clinical significance of vulnerable plaques in peripheral arteries is unclear. In the infra-inguinal circulation, plaque rupture with subsequent thrombosis has been described; however, acute presentations of this phenomenon are difficult to document in clinical practice [8]. The large caliber of the peripheral vessels, the presence of collateral channels, and the chronic nature of this disease make any acute thrombotic event difficult to ascribe to a defined plaque rupture leading to arterial occlusion. Repeated plaque ruptures and intra-atheroma hemorrhage, however, are important in the pathophysiology of coronary atheroma growth, and one might hypothesize that similar events could lead to lesion progression in the periphery [9]. The importance of vulnerable/ruptured plaques in the femoral circulation may lie in identification of individuals who are at high risk for cardiovascular events. Symptomatic PAD is associated with the presence of coronary artery disease and generally portends a very poor prognosis. Furthermore, peripheral plaques that demonstrate inflammation or are particularly lipid rich appear to be most associated with cardiac events [7]. The findings of Okura et al. of increased events (MACCE and PAD events) in patients with plaque rupture are concordant with these data.

Interestingly, the authors did not note a difference in Creactive protein (CRP) levels in patients with versus those without plaque rupture, although all of these patients had very advanced disease. While inflammation appears to play an important role in the pathophysiology of PAD, the exact role of specific biomarkers such as CRP remains unclear. Elevated CRP levels have been associated with the development and progression of PAD, with decreased future functional capacity, and poorer response to revascularization [10]. The precise role of CRP in modulating peripheral artery plaque rupture remains to be further elucidated.

The use of IVUS in the coronary arteries has provided valuable information related to arterial remodeling and vulnerable plaque physiology [3]. In contrast, plaque morphology and lesion vulnerability in the periphery has been less rigorously studied. Multiple studies have described peripheral plaque characteristics using traditional grayscale IVUS imaging. Much like the coronary circulation, such analyses are limited by the poor tissue differentiation of specific plaque components. Specifically, IVUS cannot differentiate a plaque rupture with overlying thrombus that smoothly fills the plaque cavity and thus 
may underestimate the true incidence. Although IVUS has excellent specificity for calcium, identification of fibrous and fatty tissue remains challenging. In this regard, ultrasound backscatter analysis algorithms such as the Virtual Histology (IVUS-VH; Volcano Corporation, Rancho Cordova, CA) technology may offer valuable information [4]. IVUS-VH has been used to study peripheral atheroma to variable degrees in the renal, carotid, and infrainguinal circulation. It is interesting to note that the authors of the present study used the 20-MHz Eagle Eye Gold (Volcano Corporation) catheter for some of their pullbacks, which provides VH data. While the VH data were not reported, the plaque composition of ruptured versus nonruptured femoral plaques remains an unsettled issue. Optical coherence tomography provides excellent resolution and may be ideally suited for evaluating plaque rupture in peripheral arteries.

Overall, the data presented by Okura et al. are novel and provide an interesting look at a phenomenon that has not been well described in the literature. The data are hypothesis generating but highlight an area that warrants further investigation.

Disclosure No potential conflicts of interest relevant to this article were reported.

Open Access This article is distributed under the terms of the Creative Commons Attribution Noncommercial License which permits any noncommercial use, distribution, and reproduction in any medium, provided the original author(s) and source are credited.

\section{References}

1. Prasad A, Tsimikas S: Candidate biomarkers for the detection of coronary plaque destabilization and rupture. Curr Atheroscler Rep 2008, 10:309-317.

2. Zaman AG, Helft G, Worthley SG, Badimon JJ: The role of plaque rupture and thrombosis in coronary artery disease. Atherosclerosis 2000, 149:251-266.

3. Nissen SE, Gurley JC, Grines CL, et al.: Intravascular ultrasound assessment of lumen size and wall morphology in normal subjects and patients with coronary artery disease. Circulation 1991, 84:1087-1099.

4. Diethrich EB, Irshad K, Reid DB: Virtual histology and color flow intravascular ultrasound in peripheral interventions. Semin Vasc Surg 2006, 19:155-162.

5. Kataoka T, Mathew V, Rubinshtein R, et al.: Association of plaque composition and vessel remodeling in atherosclerotic renal artery stenosis: a comparison with coronary artery disease. JACC Cardiovasc Imaging 2009, 2:327-338.

6. Martin AJ, Ryan LK, Gotlieb AI, et al.: Arterial imaging: comparison of high-resolution US and MR imaging with histologic correlation. Radiographics 1997, 17:189-202.

7. Pasterkamp G, Schoneveld AH, van der Wal AC, et al.: Inflammation of the atherosclerotic cap and shoulder of the plaque is a common and locally observed feature in unruptured plaques of femoral and coronary arteries. Arterioscler Thromb Vasc Biol 1999, 19:54-58.

8. Kohno H, Sueda S: Rupture of a peripheral popliteal artery plaque documented by intravascular ultrasound: a case report. Catheter Cardiovasc Interv 2009, 74:1102-1106.

9. Burke AP, Kolodgie FD, Farb A, et al.: Healed plaque ruptures and sudden coronary death: evidence that subclinical rupture has a role in plaque progression. Circulation 2001, 103:934-940.

10. McDermott MM, Lloyd-Jones DM: The role of biomarkers and genetics in peripheral arterial disease. J Am Coll Cardiol 2009, $54: 1228-1237$. 